\title{
ANÁLISE DE ERROS PARA ALINHAMENTO EM AHRS - PARTE II: ALGORITMOS EBA, FQA E AQUA *
}

\author{
Lucas Pimenta Silva Paiva* Rogério Menezes Filho* \\ Fidelis Marcio Santos Rabelo de Melo Júnior* \\ Leonardo Alves Vieira* Felipe Oliveira e Silva* \\ * Departamento de Automática, Universidade Federal de Lavras, MG, \\ (e-mails: paiva.lucasps@gmail.com, rogeriofilho03@gmail.com, \\ fjunyn@gmail.com, laviera1992@hotmail.com,felipe.oliveira@ufla.br).
}

\begin{abstract}
This paper proposes an error analysis of three alignment algorithms traditionally deployed in Attitude and Heading Reference Systems (AHRS): the Euler angle-Based Algorithm (EBA), the Factorial Quaternion Algorithm (FQA), and the Algebraic Quaternion Algorithm (AQUA). It is clear from the literature, that most of the comparative work between these methods has been limited to numerical rather than analytical results. Therefore, the main contribution of this work is the analytical description of the residual errors present in the computed rotation matrices (normality, orthogonality and alignment errors) and Euler angles, as well as the performance comparative study between these algorithms. In this work, the AHRS is considered a low(automotive)-grade device. Biases in the measurements of the accelerometers and magnetometers are taken into account, as well as the uncertainties in the local gravitational and magnetic fields. Simulated results confirm the proposed error analysis, evidencing the main differences, advantages and drawbacks of the use of each algorithm. Hence, this work serves as a basis for future research, especially those devoted to autonomous navigation using low-cost sensors.

Resumo: Neste artigo é realizada a análise de erros de três algoritmos de alinhamento em Sistemas de Referência de Orientação e Rumo (AHRS), a saber: o Algoritmo Baseado em Ângulos de Euler (EBA), o Algoritmo Quaternion Fatorado (FQA), e o Algoritmo Quaternion Algébrico (AQUA). Percebe-se, na literatura, que a maioria dos trabalhos comparativos entre os métodos supracitados se limita a resultados numéricos, e não analíticos. Diante disso, este trabalho tem como principal contribuição, a derivação analítica dos erros residuais desenvolvidos nas matrizes de rotação (erros de normalidade, ortogonalidade e alinhamento) e nos ângulos de Euler computados, bem como o estudo comparativo do desempenho destes algoritmos. Neste trabalho, é considerado o uso de um AHRS de nível automotivo. São considerados os biases nas medições dos acelerômetros e magnetômetros, além de incertezas nos campos gravitacional e magnético locais. Resultados simulados corroboram a análise de erros proposta, evidenciando as principais diferenças, vantagens e desvantagens da utilização de cada algoritmo. Dessa forma, este trabalho serve como base para futuras pesquisas, principalmente as que objetivam a navegação autônoma utilizando sensores de baixo custo.
\end{abstract}

Keywords: Navigation System; Alignment; AHRS.

Palavras-chaves: Sistemas de Navegação; Alinhamento; AHRS.

\section{INTRODUÇÃO}

O procedimento de alinhamento em Sistemas de Navegação Inercial (INS) é utilizado na determinação da orientação do veículo na sua etapa de inicialização (Silva et al., 2018). Segundo Groves (2008), o alinhamento consiste na determinação da matriz de rotação inicial, que relaciona o

\footnotetext{
* Esta pesquisa é financiada pela CAPES (Coordenação de Aperfeiçoamento de Pessoal de Nível Superior)(processo № 88881.169927/2018-01), Embrapa (processo № 212-20/2018) e Universidade Federal de Lavras (UFLA).
}

sistema de coordenadas da plataforma com o sistema de navegação local (norte, leste e down).

Em um INS solidário, do inglês strapdown (SINS), é importante que o processo de alinhamento seja implementado de forma autônoma, com exatidão e rapidez (Silva et al., 2018). Quando estacionário, o alinhamento é dividido em duas fases: o alinhamento grosseiro e o alinhamento fino. De acordo com Silva et al. (2018), o alinhamento grosseiro, como o próprio nome sugere, produz uma estimativa grosseira da orientação inicial do sistema, por meio de transformações analíticas bastante diretas. Os autores ainda argumentam que as principais limitações do alinhamento 
grosseiro relacionam-se às fracas suposições de sensores inerciais não-corrompidos e sistema perfeitamente estacionário.

O auto-alinhamento grosseiro de SINS é implementado a partir da observação de grandezas físicas, como a aceleração da gravidade (via acelerômetros) e a velocidade de rotação da Terra (via girômetros) (Groves, 2008). O alinhamento fino, por sua vez, melhora as estimativas fornecidas pela etapa anterior, compensando os biases dos sensores supracitados por meio de filtragem estocástica, via por exemplo, um filtro de Kalman.

No entanto, uma vez que os girômetros de baixo custo têm altas taxas de deriva e ruído, eles não são capazes de medir a velocidade angular da Terra, e portanto, não são adequados para o procedimento de alinhamento (Groves, 2008). A taxa de rotação da Terra é aproximadamente $15^{\circ} / h$, ao passo que os níveis de ruído dos girômetros de baixo custo são, em geral, dezenas de vezes maiores que este valor.

Uma solução para o procedimento de alinhamento em SINS de baixo custo consiste em se utilizar os chamados magnetômetros. Quando magnetômetros compõem uma Unidade de Medição Inercial (IMU), essa passa a ser conhecida como Sistema de Referência de Orientação e Rumo (AHRS).

Um dos primeiros algoritmos de alinhamento em AHRS foi o TRIAD (Determinação da Orientação via Três Eixos), originalmente proposto por (Shuster and Oh, 1981). Algum tempo depois, Stirling et al. (2005) propuseram um novo método para a determinação do ângulo de guinada, baseado em magnetômetros. Este método, na verdade, consiste em uma adaptação do método O-TRIAD, proposto originalmente por Jiang (1998), para SINS.

Outro método utilizado no alinhamento de AHRS é o Algoritmo Baseado em Ângulos de Euler (EBA), também conhecido como solução de orientação via arco-tangente. Este método é citado nos trabalhos de Zhu et al. (2007) e Fan et al. (2018), e, diferentemente dos demais algoritmos, fornece a solução de orientação na forma de ângulos de Euler. Conforme analisado por Groves, contudo, esta abordagem apresenta problemas de singularidade, especialmente em regiões de altas latitudes.

A fim de se evitar os problemas de singularidades provenientes do EBA, alguns autores preferem representar a orientação do veículo via quaternions, como é o caso do algoritmo QUEST (QUaternion ESTimator) (Kuga and Carrara, 2013). A principal desvantagem deste método, contudo, está ligada à sua sensibilidade à medição da densidade do campo magnético terrestre, a qual, além de corromper o ângulo de guinada, também afeta os ângulos de arfagem e rolamento.

Diante disso, Yun et al. (2008) propuseram outro algoritmo utilizando quaternions, que ficou conhecido como Algoritmo Quaternion Fatorado (FQA). Derivado do FQA, o Algoritmo Quaternion Algébrico (AQUA) foi proposto por (Valenti et al., 2016). Segundo os autores, ao contrário do FQA, o AQUA determina a orientação do veículo com base em uma seqüência de apenas duas rotações, o que reduz seu custo computacional e o torna mais eficiente.
Considerando a eficácia dos algoritmos supracitados, a maioria dos trabalhos comparativos disponíveis na literatura se limita a resultados numéricos e não analíticos. Dessa forma, este trabalho tem como principal objetivo, a derivação de expressões analíticas para os erros desenvolvidos nos algoritmos de alinhamento EBA, FQA e AQUA. $\mathrm{O}$ trabalho discute e apresenta as fórmulas para os erros de normalidade, ortogonalidade, alinhamento e ângulos de Euler, para cada um dos métodos supracitados.

O restante deste trabalho e dividido da seguinte forma: na Seção 2, são desritos os algoritmos de alinhamento em AHRS. Na Seção 3, é introduzida a análise dos erros. $\mathrm{Na}$ Seção 4, são discutidos e apresentados os resultados simulados que corroboram a análise de erros proposta. Por fim, as conclusões são apresentada na Seção 5.

\section{ALINHAMENTO EM AHRS}

\subsection{Algoritmo Baseado em Ângulos de Euler (EBA)}

O EBA determina os ângulos de rolamento $(\phi)$, arfagem $(\theta)$, e guinada $(\psi)$, a partir das seguintes igualdades,

$$
\begin{aligned}
\mathbf{g}_{P}^{b} & =C_{l}^{b} \mathbf{g}_{P}^{l}, \\
\mathbf{m}_{E}^{b} & =C_{l}^{b} \mathbf{m}_{E}^{l},
\end{aligned}
$$

com,

$$
C_{l}^{b}=\left[\begin{array}{ccc}
c \theta c \psi & c \theta s \psi & -s \theta \\
-c \phi s \psi+s \phi s \theta c \psi & c \phi c \psi+s \phi s \theta s \psi & s \phi c \theta \\
s \phi s \psi+c \phi s \theta c \psi & -s \phi c \psi+c \phi s \theta s \psi & c \phi c \theta
\end{array}\right]
$$

onde $\mathbf{g}_{P}^{l}$ é o vetor gravidade local, $\mathbf{m}_{E}$ é o vetor densidade de campo magnético terrestre, $C_{l}^{b}$ é a matriz de rotação que relaciona os triedros de navegação $(l)$ e do corpo $(b)$, e $s()$ e $c()$ representam seno e cosseno, respectivamente.

Substituindo-se (3) em (1), pode-se reescrever (para detalhes, vide Paiva et al. (2019)),

$$
\left[\begin{array}{lll}
a_{x} & a_{y} & a_{z}
\end{array}\right]^{T}=[s \theta-s \phi c \theta-c \phi c \theta]^{T} g_{P}
$$

onde $a_{x}, a_{y}$ e $a_{z}$ são os valores medidos nos três eixos dos acelerômetros, e $g_{P}$ é a magnitude do vetor gravidade local.

Manipulando-se (4),

$$
\begin{gathered}
\phi=\operatorname{atan}_{2}\left(-a_{y} /-a_{z}\right), \\
\theta=\operatorname{atan}_{2}\left(a_{x} / g_{P}\right) .
\end{gathered}
$$

A partir de (5) e (6), nota-se que a determinação de $\phi$ e $\theta$ depende unicamente das leituras dos acelerômetros e da gravidade local. Destaca-se que o valor do ângulo de arfagem poderia ser calculado sem o valor de gravidade, uma vez que esta pode ser inferida com base nos valores de $a_{y}$ e $a_{z}$. No entanto, $g_{P}$ é utilizado aqui uma vez que sua estimativa, baseada em modelos globais, é em geral, mais precisa do que a obtida com base nas saídas dos acelerômetros.

Para a determinação de $\psi$, é necessária a utilização dos magnetômetros. Substituindo-se (3) em (2), pode-se reescrever (para detalhes, vide Paiva et al. (2019)),

$$
\left[\begin{array}{l}
m_{x} \\
m_{y} \\
m_{z}
\end{array}\right]=\left[\begin{array}{rrr}
c \theta & 0 & -s \theta \\
s \phi s \theta & -c \phi & s \phi c \theta \\
c \phi s \theta & s \phi & c \phi c \theta
\end{array}\right]\left[\begin{array}{r}
c \psi_{m} c \gamma \\
s \psi_{m} c \gamma \\
s \gamma
\end{array}\right]
$$


com,

$$
\psi_{m}=\psi-\alpha
$$

onde $m_{x}, m_{y}$ e $m_{z}$ são os valores medidos nos eixos dos magnetômetros, $\alpha$ e $\gamma$ são ângulos de declinação e inclinação local do campo magnético da Terra, respectivamente, e $\psi_{m}$ é o ângulo de guinada com relação ao norte magnético.

A solução genérica para $\psi_{m}$, derivada de Groves (2008), pode ser descrita conforme,

$$
\psi_{m}=\operatorname{atan}_{2} \frac{-m_{y} c \phi+m_{z} s \phi}{m_{x} c \theta+m_{y} s \phi s \theta+m_{z} c \phi s \theta} .
$$

Substituindo-se (5) e (6) em (9), pode-se obter (Bekir, 2007),

$$
\psi_{m}=\operatorname{atan}_{2} \frac{g_{P}\left(a_{z} m_{y}-m_{y} a_{z}\right)}{a_{y}\left(a_{y} m_{x}-m_{y} a_{x}\right)-a_{z}\left(a_{x} m_{z}-m_{x} a_{z}\right)} .
$$

Uma vez que $\alpha$ é um valor conhecido, $\psi$ pode ser determinado substituindo-se (10) em (8). Para obtenção da matriz de rotação correspondente, basta substituir os valores de $\phi, \theta$ e $\psi$ diretamente em (3).

\subsection{Algoritmo Quaternion Fatorado (FQA)}

Conforme introduzido na Seção 1, o FQA foi proposto por Yun, Bachmann e Mcghee (2008), com vistas à estimação da orientação de um corpo rígido em movimento lento ou estacionário. O FQA utiliza observações da gravidade e do campo magnético da Terra, não sendo contudo, aplicável em situações onde a aceleração linear é relativamente grande, a menos que seja utilizado um filtro complementar. Este método é muito parecido com o EBA, divergindo, contudo, na representação da orientação via quaternions.

Seja o quaternion $q_{\theta}$, relativo à rotação do veículo sobre o eixo $y$ do sistema de de navegação local. Yun et al. (2008) definem $q_{\theta}$ como,

$$
q_{\theta}=c(\theta / 2)\left(\begin{array}{llll}
1 & 0 & 0 & 0
\end{array}\right)+s(\theta / 2)\left(\begin{array}{llll}
0 & 0 & 1 & 0
\end{array}\right),
$$

onde,

$$
\begin{gathered}
s(\theta / 2)=\operatorname{sign}(s \theta) \sqrt{(1-c \theta) / 2}, \\
c(\theta / 2)=\sqrt{(1+c \theta) / 2} .
\end{gathered}
$$

A fim de se evitar maior custo computacional, Yun et al. (2008) computam,

$$
\begin{gathered}
s \theta=\bar{a}_{x}, \\
c \theta=\sqrt{1-s^{2} \theta},
\end{gathered}
$$

com,

$$
\overline{\mathbf{a}}_{S F}^{b}=\left[\begin{array}{lll}
\bar{a}_{x} & \bar{a}_{y} & \bar{a}_{z}
\end{array}\right]^{T},
$$

onde $\overline{\mathbf{a}}_{S F}^{b}$ é o vetor força específica medido pelos acelerômetros, normalizado.

A determinação do quaternion $q_{\phi}$, relativo ao ângulo de rolamento, segue o mesmo princípio de $q_{\theta}$, ou seja,

$$
q_{\phi}=c(\phi / 2)\left(\begin{array}{llll}
1 & 0 & 0 & 0
\end{array}\right)+s(\phi / 2)\left(\begin{array}{llll}
0 & 1 & 0 & 0
\end{array}\right),
$$

onde $c(\phi / 2)$ e $s(\phi / 2)$ são calculados conforme (12) e (13) e,

$$
s \phi=-\left(\bar{a}_{y} / c \theta\right)
$$

$$
c \phi=\left(\bar{a}_{z} / c \theta\right) .
$$

O quaternion $q_{\psi}$, relativo ao ângulo de guinada, pode ser definido como,

$$
q_{\psi}=c(\psi / 2)\left(\begin{array}{llll}
1 & 0 & 0 & 0
\end{array}\right)+s(\psi / 2)\left(\begin{array}{llll}
0 & 0 & 0 & 1
\end{array}\right),
$$

onde $c(\psi / 2)$ e $s(\psi / 2)$ também são calculados conforme (12) e (13). Os valores de $c \psi$ e $s \psi$, por sua vez, são encontrados como segue,

$$
\left[\begin{array}{c}
c \psi \\
s \psi
\end{array}\right]=\left[\begin{array}{rr}
M_{x} & M_{y} \\
-M_{y} & M_{x}
\end{array}\right]\left[\begin{array}{l}
N_{x} \\
N_{y}
\end{array}\right],
$$

onde $N=\left[\begin{array}{ll}N_{x} & N_{y}\end{array}\right]^{T}$ é a densidade do campo magnético da Terra normalizado no plano horizontal, e $M=\left[M_{x} M_{y}\right]^{T}$ é a grandeza correspondente medida pelos magnetômetros. Estes valores podem ser calculados como,

$$
\begin{gathered}
N=\frac{1}{\sqrt{\left(m_{N}^{2}\right)+\left(m_{E}^{2}\right)}}\left[\begin{array}{c}
m_{N} \\
m_{E}
\end{array}\right], \\
M=\frac{1}{\sqrt{\left({ }^{e} m_{x}^{2}\right)+\left({ }^{e} m_{y}^{2}\right)}}\left[\begin{array}{c}
{ }^{e} m_{x} \\
{ }^{e} m_{y}
\end{array}\right],
\end{gathered}
$$

onde $m_{N}$ e $m_{E}$ são as componentes norte e leste de $\mathbf{m}_{E}^{l}$, e ${ }^{e} m_{x}$ e ${ }^{e} m_{y}$ são o segundo e terceiro componentes do quaternion intermediário ${ }^{e} m_{m}$, computado como,

$$
{ }^{e} m_{m}=q_{\theta} q_{\phi}\left({ }^{b} m_{m}\right) q_{\phi}^{-1} q_{\theta}^{-1}
$$

com,

$$
{ }^{b} m_{m}=\left(\begin{array}{llll}
0 & m_{x} & m_{y} & m_{z}
\end{array}\right)
$$

A forma final do quaternion que representa a orientação do corpo com relação ao sistema de navegação local pode ser descrita como (Yun et al., 2008),

$$
q_{b}^{l}=q_{\theta} q_{\phi} q_{\psi} \text {. }
$$

A matriz de rotação correspondente a $q_{b}^{l}$ pode ser obtida fazendo-se,

$C_{b}^{l}=\left[\begin{array}{ccc}a^{2}+b^{2}-c^{2}-d^{2} & 2(b c-a d) & 2(b d+a c) \\ 2(b c+a d) & a^{2}-b^{2}+c^{2}-d^{2} & 2(c d-a b) \\ 2(b d-a c) & 2(c d-a b) & a^{2}-b^{2}-c^{2}+d^{2}\end{array}\right]$

onde $a, b, c$ e $d$ são o primeiro, segundo, terceiro e quarto componentes de $q_{b}^{l}$, respectivamente.

Caso o resultado deva ser convertido na forma de ângulos de Euler, basta fazer,

$$
\begin{gathered}
\phi=\operatorname{atan}_{2}\left(C_{32} / C_{33}\right), \\
\theta=-\operatorname{asin}_{31}, \\
\psi=\operatorname{atan}_{2}\left(C_{21} / C_{11}\right),
\end{gathered}
$$

onde $C_{i j}$ é o elemento de $C_{b}^{l}$ na linha $i$, coluna $j$.

\subsection{Algoritmo Quaternion Algébrico (AQUA)}

Conforme introduzido na Seção 1, o algoritmo AQUA foi proposto por Valenti et al. (2016), com vistas à melhoria do desempenho dos algoritmos QUEST e FQA. Segundo Valenti et al. (2016), este agoritmo gera apenas dois quaternions: o primeiro corresponde aos ângulos de rolamento e arfagem conjuntamente, enquanto o segundo representa o movimento de guinada (Valenti et al., 2016). 
Segundo Valenti et al. (2016), no entanto, não é possível determinar tais quaternions, caso haja divergência entre as leituras dos sensores (acelerômetros e magnetômetros). Isso ocorre devido a ruidos presentes no sistema. Sendo assim, os autores sugerem o uso do triedro $g$, no lugar do triedro $l$. O triedro $g$ é uma adaptação do sistema de navegação local (NED), onde este é realinhado com o norte magnético, alterando assim seus eixos $x$ e $y$ originais. O eixo $x$ do triedro $g$, portanto, aponta na direção da projeção horizontal do campo magnético terrestre, sendo um valor não negativo, enquanto o eixo $z$ permanece na direção vertical.

Sejam, portanto, as seguintes relações,

$$
\begin{gathered}
C_{g}^{b} \overline{\mathbf{g}}_{P}^{g}=-\overline{\mathbf{a}}_{S F}^{b}, \\
C_{g}^{b} \overline{\mathbf{m}}_{E}^{g}=\overline{\mathbf{m}}_{m}^{b},
\end{gathered}
$$

onde $C_{g}^{b}$ é a matriz de rotação do recém-modificado triedro $g$ para o triedro $b$, e $\overline{\mathbf{m}}_{m}^{b}$ é o vetor densidade de fluxo magnético medido pelos magnetômetros, normalizado.

Segundo Valenti et al. (2016), são formadas duas matrizes de rotação, $C_{a c c}$ e $C_{m a g}$, correspondentes, respectivamente, aos quaternions $q_{a c c}$ e $q_{m a g}$, tal que,

$$
\begin{gathered}
C_{g}^{b}=C_{a c c} \\
C_{\text {mag }}, \\
q_{a c c}=\left[\begin{array}{llll}
q_{0 a c c} & q_{1 a c c} & q_{2 a c c} & q_{3 a c c}
\end{array}\right], \\
q_{\text {mag }}=\left[\begin{array}{llll}
q_{0 \mathrm{mag}} & 0 & 0 & q_{3 \mathrm{mag}}
\end{array}\right] .
\end{gathered}
$$

Para definição de $q_{a c c}$ é importante considerar que qualquer rotação em torno do eixo $z$ do triedro $g$, em (31), não produz alteração no resultado. Portanto, (31) é equivalente $\mathrm{a}$,

$$
C_{a c c}\left[\begin{array}{l}
0 \\
0 \\
1
\end{array}\right]=\left[\begin{array}{l}
-\bar{a}_{x} \\
-\bar{a}_{y} \\
-\bar{a}_{z}
\end{array}\right] .
$$

Após rearranjo dos termos,

$$
\left\{\begin{array}{l}
2 q_{0 a c c} q_{2 a c c}=-\bar{a}_{x} ; \\
-2 q_{0 a c c} q_{1 a c c}=-\bar{a}_{y} ; \\
q_{0 a c c}{ }^{2}-q_{1 a c c}-q_{2 a c c}{ }^{2}=-\bar{a}_{z} .
\end{array}\right.
$$

O sistema de equações em (37) permite quatro soluções para $q_{a c c}$. Segundo Valenti et al. (2016), duas destas podem ser descartadas, uma vez que possuem forma negativa. As outras duas soluções seguem as condições $q_{2 a c c}=0$, para $\bar{a}_{z} \leq 0$, e $q_{3 a c c}=0$, para $\bar{a}_{z}>0$. Dessa forma tem-se,

$$
q_{a c c}=\left\{\begin{array}{l}
{\left[\lambda_{1} \frac{\bar{a}_{y}}{2 \lambda_{1}}-\frac{\bar{a}_{x}}{2 \lambda_{1}} 0\right], \bar{a}_{z} \leq 0} \\
{\left[\frac{\bar{a}_{y}}{2 \lambda_{2}} \lambda_{2} 0-\frac{\bar{a}_{x}}{2 \lambda_{2}}\right], \bar{a}_{z}>0}
\end{array}\right.
$$

onde,

$$
\begin{aligned}
& \lambda_{1}=\sqrt{\frac{1-\bar{a}_{z}}{2}}, \\
& \lambda_{2}=\sqrt{\frac{1+\bar{a}_{z}}{2}} .
\end{aligned}
$$

Para determinar o valor de $q_{m a g}$, utiliza-se a mesma abordagem descrita acima, para $q_{a c c}$. Substituindo-se valores em (32), tem-se,

$$
C_{m a g}\left[\begin{array}{l}
L_{x} \\
L_{y} \\
L_{z}
\end{array}\right]=\left[\begin{array}{r}
\sqrt{\Gamma} \\
0 \\
L_{z}
\end{array}\right]
$$

com,

$$
\begin{gathered}
\mathbf{L}=\left[\begin{array}{l}
L_{x} \\
L_{y} \\
L_{z}
\end{array}\right]=C_{a c c}^{T}\left(\overline{\mathbf{m}}_{m}^{b}\right), \\
\Gamma=L_{x}^{2}+L_{y}^{2}
\end{gathered}
$$

onde $\mathbf{L}$ é o vetor de campo magnético rotacionado, e $C_{a c c}$ é calculado a partir de $q_{a c c}$, via (27).

Segundo Valenti et al. (2016), $q_{m a g}$ realiza apenas uma rotação sobre eixo $z$ do triedro $g$, alinhando o eixo $x$ do sistema de coordenadas intermediário, com a direção positiva do norte magnético terrestre. Essa rotação altera apenas o componente de guinada, e não afeta os ângulos de rolamento e arfagem.

Consequentemente, a partir de (41), pode-se obter o sistema,

$$
\left\{\begin{array}{l}
\left(q_{0 \mathrm{mag}}^{2}-q_{0 \mathrm{mag}}^{2}\right) \sqrt{\Gamma}=L_{x} \\
2 q_{0 \mathrm{mag}} q_{3 \mathrm{mag}} \sqrt{\Gamma}=L_{y} \\
\left(q_{0 \mathrm{mag}}^{2}+q_{3 \mathrm{mag}}^{2}\right) L_{z}=L_{z}
\end{array}\right.
$$

A fim de se evitar singularidades em (44), Valenti et al. (2016) estebelecem condições para o quaternion $q_{m a g}$, sendo elas,

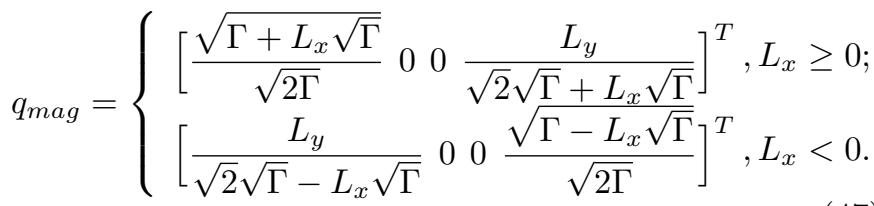

Finalmente, é possível obter o quaternion $q_{g}^{b}$, multiplicandose os dois quaternions $q_{a c c}$ e $q_{m a g}$, como segue,

$$
q_{g}^{b}=q_{a c c} q_{m a g}
$$

\section{ANÁLISE DE ERROS}

A respeito da validade das equações apresentadas na Seção 2, elas se basearam nas seguintes premissas: veículo perfeitamente estacionário, sensores não corrompidos e as condições externas (gravidade e campo magnético terrestre) perfeitamente conhecidos.

A existência de erros nas variáveis acima descritas origina matrizez de rotação corrompidas, $\hat{C}_{b}^{l}$, bem como ângulos de Euler corrompidos, $\hat{\phi}, \hat{\theta}$ e $\hat{\psi}$. Neste trabalho, os erros em $\hat{C}_{b}^{l}$ são descritos na forma de erros de normalidade, $\eta^{l}$, ortogonalidade, $\mathbf{o}^{l}$, e alinhamento, $\varphi^{l}$, e foram derivados seguindo a metodologia proposta por (Paiva et al., 2019).

Ainda de acordo como Paiva et al. (2019), as expressões analíticas obtidas para os erros supracitados, para o caso genérico de um veículo arbitrariamente orientado com relação ao sistema de navegação, são complexas e de difícil interpletação. Sendo assim, o autor adota a condição de perfeito alinhamento entre os triedros do corpo e de navegação, condição essa que também é adotada neste trabalho.

\subsection{EBA}

Procedendo-se com a análise de erros para o algoritmo EBA, via metodologia proposta em Paiva et al. (2019), obtêm-se as seguintes expressões analíticas, 


$$
\begin{gathered}
\eta_{N}=\eta_{E}=\eta_{D}=o_{N}=o_{E}=o_{D}=0, \\
\varphi_{N}=-\delta \phi=-\frac{1}{g_{P}} \delta a_{x}, \\
\varphi_{E}=-\delta \theta=\frac{1}{g_{P}} \delta a_{x}, \\
\varphi_{D}=-\delta \psi=\frac{s \alpha t \gamma}{g_{P}} \delta a_{x}+\frac{c \alpha t a n \gamma}{g_{P}} \delta a_{y} \\
-\frac{s \alpha}{B c \gamma} \delta m_{x}+\frac{c \alpha}{B c \gamma} \delta m_{y}-\delta \alpha,
\end{gathered}
$$

onde $B$ é a magnitude da densidade de campo magnético terrestre, e $\delta_{x}$ representa o erro na variável genérica $x$.

É possível perceber que, diferentemente do algoritmo TRIAD, investigado por Paiva et al. (2019), o algoritmo EBA elimina os erros de normalidade e ortogonalidade, reduzindo assim o custo computacional de implementação do algoritmo. Os erros desenvolvidos no EBA são exclusivamente erros de alinhamento e nos ângulos de Euler. Observa-se também, que os erros de alinhamento norte, leste e down são iguais em magnitude e opostos em sinal aos erros de rolamento, arfagem e guinada, respectivamente.

Adicionalmente, verifica-se em (50) e (51) que, diferentemente da TRIAD, os erros de alinhamento norte e leste no EBA não são afetados por erros nos magnetômetros, ou no campo magnético terrestre.

\subsection{FQA e AQUA}

Os métodos FQA e AQUA utilizam representacões de atitude na forma quaternions. Expressões analíticas para os erros provenientes destes métodos podem ser derivadas convertendo-se primeiramente os quaternions em correspondentes matrizes de rotação, via (27), e empregando-se, a seguir, a metodologia proposta por Paiva et al. (2019).

Procedendo de tal maneira, encontram-se exatamente as mesmas expressões analíticas obtidas para o algoritmo EBA. Isso confirma o trabalho de Yun et al. (2008), no qual o desempenho dos algoritmos EBA e FQA foi dito ser semelhante.

Dessa forma, pode-se concluir que o FQA e o AQUA, assim como o EBA, são superiores ao TRIAD, analisado em Paiva et al. (2019). Na realidade, pode-se considerar o FQA e AQUA superiores ao EBA, uma vez que eles evitam problemas de singularidade, bem como o cálculo de funções trigonométricas Yun et al. (2008).

Do ponto de vista de eficiência computacional, Valenti et al. (2016), ainda concluem que o AQUA pode ser considerado superior ao FQA, pois o procedimento para determinação do ângulo de guinada, no AQUA, elimina a necessidade da pré-definição do campo magnético.

\section{RESULTADOS SIMULADOS}

Para validar a análise de erros apresentada na Seção 3 , bem como as verificações apresentadas, foi elaborado um teste simulado.

Os resultados se baseiam no teste de desempenho do processo de alinhamento usando os algoritmos EBA, FQA e
AQUA. Para fins de comparação, também foi incluído na análise o algoritmo TRIAD (vide Paiva et al. (2019), para detalhes). Nesta simulação, foram considerados valores de latitude local $-23^{\circ} 12^{\prime} 47^{\prime \prime}$, longitude $-45^{\circ} 51^{\prime} 38^{\prime \prime}$ e altitude 629 metros. Os dados da Terra, como raio equatorial, elipticidade, velocidade ângular, entre outros, foram baseados no trabalho de Rogers (2007) apud Silva (2016). A aceleração da gravidade local e o campo magnético da Terra foram calculados de acordo com os modelos descritos em Britting (1971) e Chulliat et al. (2015), respectivamente.

Por definição, as simulações foram concebidas assumindose que o sistema de coordenadas do corpo se encontra perfeitamente alinhado com o sistema de navegação local, representando, portanto, um cenário ideal.

Os sensores (acelerômetros e magnetômetros) foram simulados estacionários com frequência de amostragem de $100 \mathrm{~Hz}$. Os biases simulados dos magnetômetros e acelerômetros foram de, respectivamente, $5 \mathrm{mG}$ e $5 \mathrm{mg}$. Também foram utilizados valores de random walk para os acelerômetros $(0.1 \mathrm{mg} / \sqrt{\mathrm{Hz}})$ e magnetômetros $(0.2 \mathrm{mG} / \sqrt{\mathrm{Hz}})$. Incertezas nos valores da gravidade local, ângulo de declinação, ângulo de inclinação, e magnitude do campo magnético local também foram consideradas, a saber, 0,005 $\mathrm{mg}, 0.1^{\circ}, 0,1^{\circ}$ e $0,1 \mathrm{mG}$, respectivamente. Esses valores simulam uma Unidade de Medição Inercial de nível automotivo conforme as especificações descritas no trabalho de Groves (2008).

Com base nos erros simulados foi possível obter estimativas para os erros de normalidade, ortogonalidade, alinhamento e ângulos de Euler para cada um dos algoritmos investigados. Esses valores são oriundos das fórmulas apresentadas na Seção 4 (deste trabalho, e de Paiva et al. (2019)), e estão representados na Tabela 1.

A Figura 1, por outro lado, ilustra os erros obtidos ao longo do processo de alinhamento. Como esperado, os métodos EBA, FQA e AQUA não resultaram em erros de ortonormalidade.

Nas Figuras 1(g), (h) e (i), é possível observar que: 1) os resultados de alinhamento dos algoritmos tem um comportamento parecido na direção down; 2) o algoritmo TRIAD apresenta menor erro na direção norte, enquanto o EBA, FQA e AQUA possuem comportamentos parecidos;

\begin{tabular}{|c|c|c|c|c|}
\hline Erros & TRIAD & EBA & FQA & AQUA \\
\hline$\eta_{N}\left[^{\circ}\right]$ & 0,6451 & 0 & 0 & 0 \\
\hline$\eta_{E}\left[^{\circ}\right]$ & 0,4235 & 0 & 0 & 0 \\
\hline$\eta_{D}\left[^{\circ}\right]$ & $-0,2873$ & 0 & 0 & 0 \\
\hline $\mathrm{o}_{N}\left[{ }^{\circ}\right]$ & $-0,2070$ & 0 & 0 & 0 \\
\hline $\mathrm{o}_{E}\left[^{\circ}\right]$ & 0,5196 & 0 & 0 & 0 \\
\hline $\mathrm{o}_{D}\left[^{\circ}\right]$ & $-0,1049$ & 0 & 0 & 0 \\
\hline$\varphi_{N}\left[{ }^{0}\right]$ & 0,0781 & 0,2862 & 0,2862 & 0,2862 \\
\hline$\varphi_{E}\left[{ }^{\circ}\right]$ & $-0,8140$ & $-0,2896$ & $-0,2910$ & $-0,2910$ \\
\hline$\varphi_{D}\left[{ }^{\circ}\right]$ & 1,6594 & 1,6484 & 1,6480 & 1,6480 \\
\hline$\left.\delta \phi \quad{ }^{\circ}\right]$ & $-0,2903$ & $-0,2903$ & $-0,2903$ & $-0,2903$ \\
\hline$\delta \theta$ & 0,2855 & 0,2855 & 0,2869 & 0,2869 \\
\hline$\delta \psi$ & $-1,7400$ & $-1,6494$ & $-1,6490$ & $-1,6490$ \\
\hline
\end{tabular}
3) o erro de alinhamento leste dos algoritmos EBA, FQA e AQUA é menor que o do TRIAD, possuindo também, menor tempo de convergência.

Tabela 1. Erros simulados. 

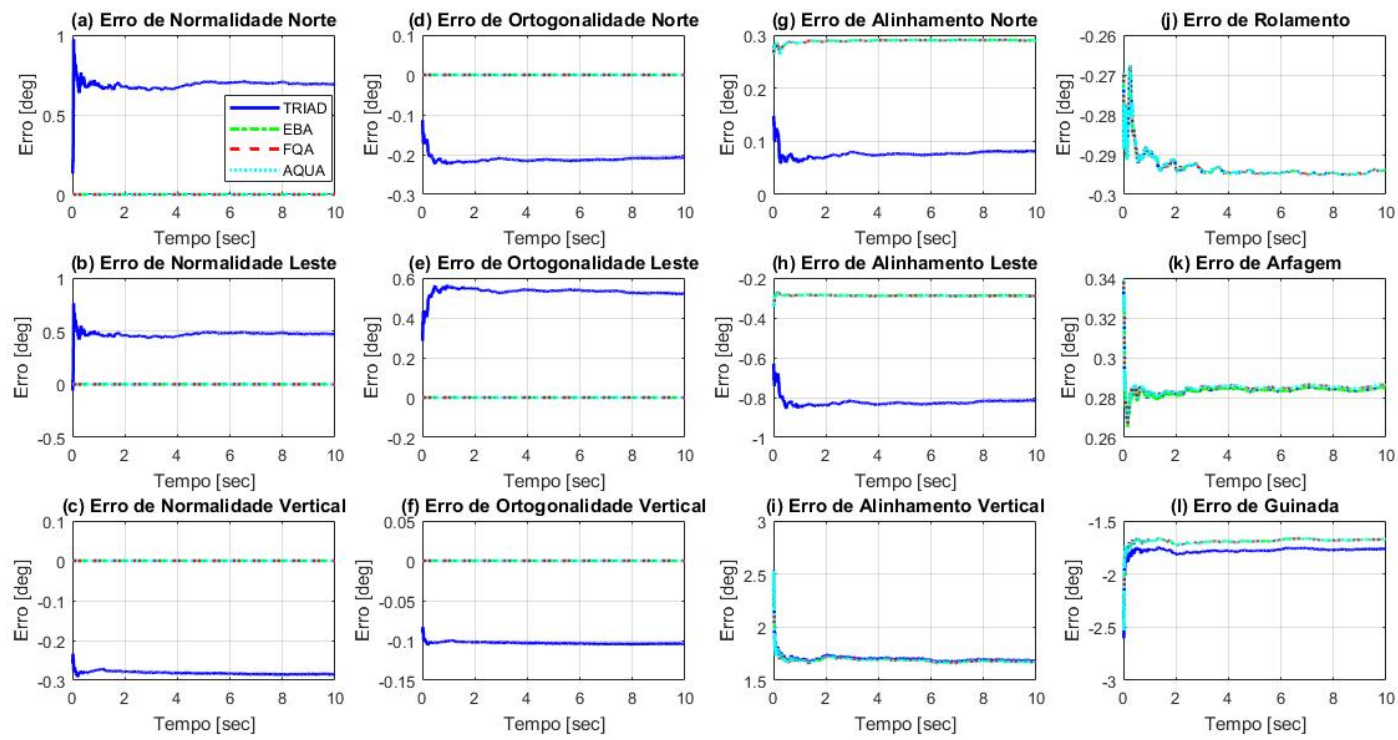

Figura 1. Erros Simulados

Fonte: Autor.

Já nas Figuras 1(j) e (1), observa-se erros de rolamento e arfagem se sobrepõem, para todos os algoritmos investigados. Como discutido na Seção 2, todos eles estimam o ângulo de rolamento e arfagem com base, apenas, nas leituras dos acelerômetros, justificando, portanto, tal semelhança.

Na Figura 1(l), por fim, nota-se que o erro residual de guinada, desenvolvido pelo algoritmo TRIAD, é ligeiramente maior ao dos algoritmos EBA, FQA e AQUA. Verificase, de forma geral, que os erros obtidos na simulação (Figura 1) corroboram os erros preditos (Tabela 1). Estes resultados, portanto confirmam a adequação da análise de erros proposta neste artigo.

Destaca-se, por fim, que os métodos EBA, FQA e AQUA originaram resultados muito próximos, do ponto de vista de exatidão. No entanto, eles diferem quanto ao custo computacional. O algoritmo AQUA, embora apresente desempenho semelhante ao EBA e o FQA, pode ser considerado mais eficiente, pois, de acordo com Valenti et al. (2016), seu custo computacioal é menor em até $65 \%$.

\section{CONCLUSÕES}

Este trabalho apresentou uma análise de erros para três algoritmos de alinhamento grosseiro empregados em Sistemas de Referência de Orientação e Rumo (AHRS), a saber: o EBA, FQA e AQUA.

Para fins de análise, foram considerados erros nas leituras dos acelerômetros e magnetômetros, bem como nos modelos gravitacional e magnético da Terra. Um cenário ideal de sistemas de coordenadas do corpo e de navegação perfeitamente alinhados foi adotado.

Expressões analíticas para os erros residuais (normalidade, ortogonalidade e alinhamento) desenvolvidos nas matrizes de rotação computadas por cada um dos algoritmos foram derivadas. Além disso, foram derivadas expressões para os erros nos correspondentes ângulos de Euler.
Como fruto da análise, verificou-se que os algoritmos se assemelham em termos de exatidão, sendo todos, contudo, superiores ao algoritmo TRIAD, investigado em Paiva et al. (2019). Embora os algoritmos EBA, FQA e AQUA apresentem desempenhos semelhantes, verifica-se, na literatura, que o algoritmo AQUA implica em menor custo computacional, podendo portanto, ser considerado mais eficiente para fins de alinhamento em AHRS.

Como metas para continuação deste trabalho, os autores pretendem expandir a análise de erros de forma mais criteriosa, tornando-a válida, por exemplo, para qualquer orientação do sistema de coordenadas do corpo com relação ao de navegação. Assim como aprofundar a discussão à respeito dos erros obtidos, em cada método, também na fase de alinhamento fino. Diferentes algoritmos, como o QUaternion ESTimator (QUEST), e o Super-fast Attitude of Accelerometer and Magnetometer (SAAM), também deverão ser implementados, como forma de comparar seus desempenhos com os demais algoritmos estudados. Por fim, os autores pretendem conduzir testes experimentais, com dados reais colhidos de uma AHRS, com vistas à validaçao dos resultados simulados aqui apresentados.

\section{AGRADECIMENTOS}

Os autores agradecem ao Departamento de Automática (DAT) da Universidade Federal de Lavras (UFLA), pelo suporte dado à pesquisa.

\section{REFERÊNCIAS}

Bekir, E. (2007). Introduction to modern navigation systems. World Scientific.

Britting, K.R. (1971). Inertial navigation systems analysis. Chulliat, A., Macmillan, S., Alken, P., Beggan, C., Nair, M., Hamilton, B., Woods, A., Ridley, V., Maus, S., and Thomson, A. (2015). The US/UK world magnetic model for 2015-2020. 
Fan, B., Li, Q., and Liu, T. (2018). How magnetic disturbance influences the attitude and heading in magnetic and inertial sensor-based orientation estimation. Sensors, 18(1), 76.

Groves (2008). Principles of GNSS, inertial, and multisensor integrated navigation systems.

Jiang, Y.F. (1998). Error analysis of analytic coarse alignment methods. IEEE Transactions on Aerospace and Electronic Systems, 34(1), 334-337.

Kuga, H.K. and Carrara, V. (2013). Attitude determination with magnetometers and accelerometers to use in satellite simulator. Mathematical Problems in Engineering, 2013.

Paiva, L.P.S., Júnior, F.M.S.R.M., Filho, R.M., Vieira, L.A., and Silva, F.O. (2019). Análise de erros para alinhamento em AHRS - parte I: Algoritmo TRIAD. In Simpósio Brasileiro de Automação Inteligente, 2019, submetido.

Shuster, M.D. and Oh, S.D. (1981). Three-axis attitude determination from vector observations. Journal of Guidance, Control, and Dynamics, 4(1), 70-77.

Silva, F.O. (2016). Stationary Self-Alignment and Calibration Techniques for Straodown Inertial Navigation Systems. Addison-Wesley.

Silva, F.O., Hemerly, E.M., Filho, W.C.L., and Kuga, H.K. (2018). A fast in-field coarse alignment and bias estimation method for stationary intermediate-grade IMUs. IEEE Transactions on Instrumentation and Measurement, 67(4), 831-838.

Stirling, R., Fyfe, K., and Lachapelle, G. (2005). Evaluation of a new method of heading estimation for pedestrian dead reckoning using shoe mounted sensors. The Journal of Navigation, 58(1), 31-45.

Valenti, R.G., Dryanovski, I., and Xiao, J. (2016). A linear Kalman filter for MARG orientation estimation using the algebraic quaternion algorithm. IEEE Transactions on Instrumentation and Measurement, 65(2), 467-481.

Yun, X., Bachmann, E.R., and McGhee, R.B. (2008). A simplified quaternion-based algorithm for orientation estimation from earth gravity and magnetic field measurements. IEEE Transactions on instrumentation and measurement, 57(3), 638-650.

Zhu, R., Sun, D., Zhou, Z., and Wang, D. (2007). A linear fusion algorithm for attitude determination using low cost mems-based sensors. Measurement, 40(3), 322-328. 